\title{
Uma aplicação do Z-Score
}

\section{An application of the Z-Score}

\author{
Bruno José Machado de Almeida ${ }^{1}$ \\ Pedro Miguel Torres Carvalho \\ José Joaquim Marques de Almeida ${ }^{3}$
}

\section{Resumo}

O propósito deste estudo é aplicar a metodologia do Z-score a uma empresa do setor metalúrgico português que opera em um ambiente generalizado de crise estrutural, o que acabou por repercutir no setor e na organização. Coligimos as demonstrações financeiras da empresa, desde 2008 até 2012, tendo coletado os dados indispensáveis à aplicação do modelo. Em simultâneo, procedemos à análise da estratégia da empresa refletida nos planos e programas previsionais de 2012 a 2015. Obtidos e analisados os indicadores do Z-score relacionados com a história da empresa, imediatamente constatámos a sua profunda degradação. No entanto, a administração da unidade, apoiada em uma estratégia de diversificação e redução de custos, sempre julgou favoravelmente a continuidade da empresa. A estratégia de turnaround encetada e apoiada em informação financeira prospectiva, baseada em pressupostos credíveis, evidencia que os indicadores do Z-score recuperavam à medida que a linha estratégica formulada ia sendo implementada. A conclusão obtida aponta para a importância da estratégia no going concern, situação que é evidenciada na quantificação proporcionada pelo modelo.

Palavras-chave: Estratégia. Going concern. Z-score. Orçamentos. Previsões.

1 Doutorado em Ciências Económicas Empresariais na área de Contabilidade e Auditoria pela Universidad Complutense de Madrid (Espanha), Mestre em Contabilidade e Auditoria pela Universidade Aberta (Portugal), Licenciado em Contabilidade e Auditoria pelo Instituto Superior de Contabilidade e Administração de Coimbra (Portugal), Professor Coordenador da Escola de Negócios de Coimbra (Coimbra Business School) - Portugal - email: brunojmalmeida@gmail. com

2 Licenciado em Organização e Gestão de Empresas pela Faculdade de Economia da Universidade de Coimbra (Portugal), MBA em Gestão de Empresas pelo Instituto Superior de Ciências do Trabalho e da Empresa (Portugal) - Portugal - email: pedrocarvalho@gesconsulting.pt

3 Doutorado em Ciências Económicas Empresariais na área de Contabilidade e Auditoria pela Universidad Complutense de Madrid (Espanha), Professor Catedrático da Universidade Lusíada (Portugal) - Portugal - email: marquesdealmeida.roc@gmail.com 


\section{Abstract}

The purpose of this study is to apply the Z-score methodology to a company in the Portuguese metallurgical sector operating in a structural crisis environment which had an impact on this sector and its organization. Thus, we gathered the financial statements of the company, from 2008 to 2012, and collected the essential data to apply the model. Simultaneously, we analysed the company's strategy reflected on the plans and forward programs from 2012 to 2015. When we obtained and analysed the Z-score indicators related to the history of the company, we immediately noticed its serious deterioration. However, the administration of the unit, based on the strategy of diversification and reduction of costs, always thought it favorable to continue with the company. The turnaround strategy initiated and supported by prospective financial information, based on credible assumptions, clearly indicates that the Z-score indicators recover alongside with the implementation of this strategic line. The conclusions obtained indicate the importance of the strategy in the going concern, which is evident in the quantification provided by the model.

Keywords: Strategy. Going concern. Z-score. Budgets. Forecasts.

\section{Introdução}

Em um contexto de instabilidade económica conjuntural e estrutural, os modelos de previsão de crises empresariais são um instrumento importante de diagnóstico, cuja aplicação tem uma função preventiva, por habilitarem a administração da empresa a tomar as decisões corretivas apropriadas à sua sobrevivência. Desse modo, interrogamo-nos se a aplicação do Z-score a um caso concreto possibilita ou não tomar um conjunto de decisões, de forma atempada, conducentes a inverter a tendência negativa dos ratios do modelo discriminante de Altman. Portanto, o objetivo geral é aplicar o modelo do Z-score a uma empresa singular do setor metalúrgico português e, em termos específicos, analisar o triângulo de sustentabilidade assente em três pilares: going concern, Z-score e estratégia.

A necessidade de obtenção, por parte do auditor externo ou interno, de um conhecimento total do negócio do cliente, da indústria, do setor, da economia do país e da economia mundial (LEWIS, 2012) direciona tendencialmente a auditoria para a estratégia, prevendo- 
se a sua utilização, cada vez mais intensa, nesse nicho específico de mercado (VENDERBECK, 2012).

Acresce que, downturn da economia mundial, a partir do ano 2000, criou-se nas organizações internacionais da auditoria - IAASB (International Auditing and Assurance Standard Board) - a consciência de desenvolver um plano ambicioso que fomentou um conjunto de novas normas internacionais de auditoria (ISA - International Standards on Auditing), focando o risco de negócio e a avaliação dos sistemas de controlo interno - ISA 240, 315, 220, 540 etc. -, o que fez emergir novas necessidades e instrumentos de análise mais aperfeiçoados para a condução da auditoria em envolvente instável. A KPMG (BELL; MARRS, 2005), na sequência da emergência da Sarbanes Oxley - SOX - (2002) e da criação do PCAOB (Public Company Accounting Oversight Board) como entidade de supervisão de auditoria nos EUA, aperfeiçoou os modelos de análise de risco. Concebeu o BMP - Business Measurement Process -, propôs modelos renovados de auditoria global - global audit methodology triangulation - e novas abordagens para o planeamento da análise do risco, com o objetivo de ganhar vantagem competitiva na chamada strategic audit, conceituada como a avaliação total dos fatores materialmente relevantes que afetam o bem-estar económico de uma empresa.

Como as demonstrações financeiras são o resultado de uma estratégia, a ligação ao going concern é um axioma indiscutível. 0 conceito pressupõe que a empresa opere ilimitadamente e que não será liquidada num futuro próximo.

Assim, quando permanecem dúvidas quanto à continuidade da empresa, a única possibilidade de evitar uma reserva por incerteza na aplicação do princípio da empresa em funcionamento é precisamente a análise da informação financeira prospectiva, facultada pela administração da entidade. A capacidade preditiva dos modelos de previsão (ALTMAN; MCGOUGH, 1974; BEAVER, 1966; ZMIJEWSKI, 1984) depende de um conjunto de ratios económico-financeiros que se estima que tenham, à partida, capacidade como preditores de falências. 
O Z-score proporciona uma informação comparativa da posição relativa da empresa relativamente à sua insolvência. Em face do exposto, torna-se evidente a interdependência da estratégia, going concern e Z-score, conforme evidenciamos na Figura 1.

Figura 1: Triângulo de sustentabilidade.

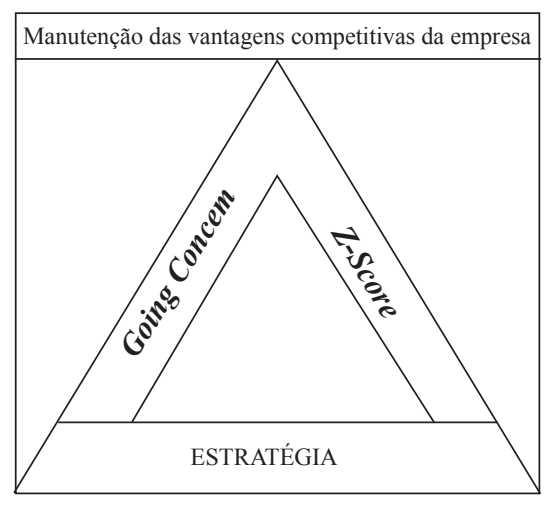

Fonte: Autor.

Apresentado o tema, na secção seguinte desenvolvemos a literatura. Na segunda parte, apresentamos a metodologia de investigação utilizada. Na terceira, evidenciamos o case study e, por fim, são retiradas as respetivas conclusões.

\section{Revisão da bibliografia}

O conhecimento cada vez mais rigoroso e tempestivo do risco do negócio, em um contexto de forte internacionalização das empresas, que ocorre desde 1975, fez emergir várias escolas de estratégia: a escola do posicionamento competitivo (PORTER, 1996), a escola da emergência (MINTZBERG, 1996) e a escola do desenvolvimento das competências distintivas (HAMEL; PRAHALAD, 1995), todas elas dotadas de objetivos em longo prazo (strategic intent).

Esse novo paradigma de abordagem do management ganhou avanço relativamente às filosofias de gestão ancoradas no primado 
da produção, que estavam estruturadas nas escolas de organização científica do trabalho (FAYOL, 1916; TAYLOR; GLEZEN, 1994) e nas escolas das relações humanas e da teoria do comportamento estratégico ou de direção estratégica.

Essas últimas escolas tinham como objetivo responder à envolvente económica existente, em que a empresa estava fechada sobre si mesma, não se preocupando em identificar as necessidades e os valores de um mercado-alvo. São escolas que assentavam em uma ideia de incrementalismo (BUENO CAMPOS, 1996).

Por sua vez, a perspetiva de Godet, aplicada nos anos 1970 às ciências sociais, dá origem à corrente do determinismo indeterminável e sugere conceitos novos, tais como: efeito borboleta, princípio de incerteza de Heisenberg etc. Por sua vez, Hawkins, Nissen e Rendon (2014) apontam para as consequências estratégicas das mudanças da envolvente, focando a necessidade de flexibilidade estratégica.

A implantação da estratégia, em um mundo em constante mutação, é objeto de análise por um conjunto amplo de autores. Porter (2008) e Lafley et al. (2012) chamam atenção para o facto de uma estratégia brilhantemente concebida ser somente uma condição necessária e não suficiente. A sua execução sólida e implementação adequada são indispensáveis para o êxito e continuidade da empresa - going concern.

Essse princípio é assumido, de uma maneira geral, como o pressuposto em que assenta a contabilidade (DICKSEE, 1905). Na mesma linha, Paton (1962) e Littleton (1966) atribuem ao conceito de continuidade a categoria de postulado básico. Outros, como ljiry (1965), Sterling (1968), contestam os argumentos da corrente do pressuposto da continuidade, observando que as estatísticas que analisam o tempo médio de vida das empresas apontam a limitação temporal como um facto corrente e indesmentível.

O pressuposto da continuidade impõe que a empresa mantenha a sua capacidade competitiva e, nesse contexto, o conceito de continuidade a ser retido deve ser interpretado preferentemente como uma previsão. 
É por isso que o órgão de gestão, para avaliar se a entidade está ou não em continuidade, deverá levar em linha de conta toda a informação sobre o futuro e considerar os 12 meses seguintes à data do balanço ( $\S$ 26 IAS 1).

Ao colocar-se a problemática da continuidade/descontinuidade no âmbito e alcance da auditoria, esta sai do seu nicho tradicional para se situar na análise da viabilidade do negócio e da lucratividade, que passariam a ser elementos fundamentais da auditoria às demonstrações financeiras. A auditoria à estratégia pressupõe uma informação de natureza previsional, situação que impõe aos auditores o domínio das técnicas de previsão suscetíveis de o apoiar no diagnóstico do going concern. De facto, a saúde financeira das entidades empresariais é um tema central para todos os stakeholders (AUCHTERLOINE, 1997).

Os primeiros modelos de previsão utilizavam a informação financeira contabilística e histórica na conceção dos modelos de previsão, por meio da extrapolação da tendência, não sendo, assim, considerados, na estrutura desses modelos, os objetivos futuros que a administração da empresa pretende alcançar. Isto é, a atuação da empresa não é uma variável considerada (MARQUES DE ALMEIDA, 2000). Beaver (1966) informa que a análise discriminante começou a ser utilizada no início do século XX pelas entidades bancárias, com o propósito de avaliar 0 chamado risco de crédito, focado no chamado indicador de liquidez geral, que indicava o grau de solvência da empresa, técnica conhecida pelo current ratio. Os modelos multivariantes - discriminantes ou modelos de probabilidade condicional logit - têm a sua origem na fórmula inicial de Altman e McGough (1974), que procura uma combinação ótica de ratios que melhor desempenhe o papel de preditor de uma falência.

No atual contexto de incerteza, os modelos de previsão de falência voltaram a despertar o interesse dos práticos e dos académicos. Assim sucede com Altman, Danovi e Falini (2013), ao aplicarem o Z-score a empresas italianas. Özdemir (2014) aplica as técnicas às empresas turcas e, por fim, Rãscolean, Dobra e Slusariuc (2012) elegem o Z-score para prognosticar a falência das empresas. 
Por sua vez, Anjum (2012) analisa a evolução histórica do modelo de Altman desde a sua formulação original (1968), passando pela sua reformulação $(1983,1993)$, e nota que o modelo tem uma taxa de sucesso de $90,9 \%$ na previsão de falências. Chao (2012) salienta que uma das características fundamentais do Z-score é a sua capacidade de utilização de informação financeira e contabilística apresentada e divulgada pelas empresas, que é agregada num conjunto de indicadores de grande importância contabilística.

\section{Metodologia}

De acordo com Kilpatrick (1998), o estudo de um caso é essencialmente um desenho de investigação no qual se incorporam paradigmas metodológicos diferenciados: o positivista e o interpretativo. O método aplicado constitui, assim, uma exploração intensiva de uma simples unidade de estudo (ERICKSON, 1986) - empresa metalúrgica -, escolhida com base na experiência pessoal do investigador (FREIXO, 2011), constituindo, assim, uma amostra por seleção natural, em que o elemento da população é escolhido com base na correspondência entre as suas características e os objetivos do estudo. De facto, pretendia-se responder à seguinte questão:

- Porque os Z-scores apontavam tendencialmente o encerramento da empresa, e os administradores, de acordo com o seu melhor conhecimento, apostavam na continuidade da empresa?

- Por que a estratégia da empresa não estava a resultar?

Ponte (2006) considera que essa metodologia pode utilizar uma grande variedade de instrumentos e estratégias, que, baseadas em uma análise documental, refletem o estudo da empresa em observação no seu contexto real. Em termos analíticos, interrogamos a situação investigando o fenómeno empiricamente (FREIXO, 2011), com definição prévia das questões de investigação e da unidade de análise (YIN, 2005), densificadas nas questões atrás enunciadas. 
Para o efeito, os dados obtidos no decurso de 2011, consistiram na recolha de toda a informação histórica da empresa e permitem-nos responder às questões: o que aconteceu e como. Os dados previsionais, por sua vez, foram obtidos em 2012 e basearam-se na análise dos programas e orçamentos previsionais, incluídos no seu programa estratégico. Estes dados - contabilísticos e previsionais - foram ajustados, ordenados e classificados para serem induzidos no modelo de Z-score. Para o efeito, elaborámos uma folha de cálculo Excel.

\section{Estudo de um caso}

\subsection{Apresentação da empresa}

Trata-se de uma empresa do setor metalúrgico cuja atividade consiste na construção metalomecânica de estruturas e tubagens, na manutenção de conservação industrial e na construção de estruturas de edifícios e equipamentos industriais. Opera em um setor em claras dificuldades: inexistência de planos para a renovação de grandes estruturas industriais, e o setor da construção enfrenta e concentra grande parte da sua produção em poucos clientes, sendo que um deles está ligado ao setor. Assim, foi opção, na fase de planeamento da auditoria, testar o Z-Score como complemento à avaliação do risco, de modo a determinar se existem fatores que possam indiciar a incapacidade da entidade para prosseguir em continuidade.

Dado que esse modelo deve ser analisado de forma contínua, de modo que seja avaliada a sua evolução, e não de forma estática, a análise foi efetuada desde o ano de 2008. Altman, Donovi e Falini (2013), Elliot, Siu e Fung (2014), Rãscolean, Dobra e Slusariuc (2012) enaltecem o Z-score como medida de avaliação da saúde financeira de uma empresa, além de o considerarem como uma fonte importante de informação para avaliar a capacidade de concessão de crédito às empresas, tomando por base os indicadores contabilísticos. O modelo foi aplicado entre o ano de 2008 e 2012, e estimado até 2015, tendo em conta as características da envolvente. 
Estando num período de recessão, o auditor deverá estar mais atento aos indicadores que possam revelar dificuldades na capacidade intrínseca da entidade em gerar resultados. Apesar de não ter sido usado o modelo nos procedimentos de auditoria do ano de 2011, existem fatores que alertaram para a possibilidade de, em 2012, a entidade poder apresentar riscos de, a médio prazo, começar a ter dificuldades. De facto, Chao (2012) refere que o modelo tem sido sistematicamente modificado para ser suscetível de aplicação às várias indústrias, mas reconhece a sua aceitação por parte dos auditores como instrumento de diagnóstico do going concern, por utilizar informação financeira que a empresa apresenta e publica.

Os indicadores relativos ao ano de 2011, o que se espera do ano de 2012 e as perspetivas que existem para o ano de 2013 e seguintes fomentaram uma maior preocupação em analisar os pressupostos de continuidade assumidos pela empresa.

Por sua vez, a previsão para o volume de negócios para o ano de 2012 foi, no início do ano, de cerca de 14.500.000€. De acordo com as estimativas, o valor final deverá ser significativamente inferior. Essa previsão teve como erro a consideração demasiado otimista de que o seu maior cliente, que representa cerca de $50 \%$ do volume de negócios, não reduzisse significativamente as suas encomendas, tendo em conta o setor em que opera (minas). Na realidade, o nível de atividade com esse cliente reduziu-se para cerca de $60 \%$ do previsto. Também o segundo cliente (cimenteira) reduziu a sua atividade significativamente, prevendo-se que a sua produção se reduza para 1/3.

Estima-se que o volume de negócios seja, no final de 2012, de cerca de $13.500 .000 €$, valor significativamente inferior ao inicialmente previsto. De acordo com a estrutura de custos existente neste momento, os resultados serão negativos. A entidade depende significativamente do recurso ao crédito, recurso que tem cada vez mais dificuldade em obter. Por outro lado, com a redução nas vendas para o maior cliente, ao qual está associado um contrato de factoring, começa a defrontar-se com problemas de liquidez e tesouraria. Em suma, a entidade debate- 
se, por um lado, com problemas de rendibilidade, por via da redução nas vendas ao cliente com mais representatividade e, por outro, com problemas financeiros.

\subsection{A estratégia desenvolvida de 2000-2008-2012}

A análise das envolventes interna e externa é considerada determinante para a formulação do plano estratégico (CĂPRIOARĂ, 2014; PORTER, 1996; LEWIS, 2012; PARASCHIVESCU), sendo, neste enquadramento, a empresa metalúrgica encarada como um organismo com objetivos próprios, inserida num sistema social mais vasto.

Por sua vez, a análise da envolvente externa permitiu à empresa detetar ameaças e oportunidades e desenvolver estratégias adaptativas indispensáveis ao sucesso e sobrevivência da organização (GRANT, 2010; HITT; IRELAND; HOSKISSON, 2007, 2009).

Além da identificação da estratégia geral e da estratégia ao nível do negócio, a estratégia funcional adotada centrou-se na maximização da produtividade, que induzia uma competência distintiva e possibilitava à empresa a obtenção de uma vantagem competitiva importante, tendo em conta a existência de prazos muito apertados na manutenção e conservação das grandes unidades industriais.

Uma vez identificada a posição atual e futura da empresa, as competências básicas distintivas foram identificadas com a produtividade e o prazo, considerados fatores importantes da manutenção da capacidade produtiva da empresa e, portanto, da sua continuidade. Selecionada essa estratégia como a melhor alternativa possível, ela foi adequadamente implementada por meio da conversão das políticas eleitas em táticas, programas e orçamentos para cada obra adjudicada. No plano de médio/longo prazo (8 anos), foram tratados fundamentalmente os recursos necessários para se atingirem os objetivos definidos. Em nível de curto prazo, a posição estratégica deve refletir-se na conta de resultados que, por sua vez, podem se segmentar por obras. Essa estratégia deu claros frutos até 2008 , ano em que as vendas atingiram 
30M€ de faturação e proporcionaram um EBITDA (Earnings before interest, taxes, depreciation, and amortization) de 1,199M€.

A partir desse ano, a crise económica afetou a capacidade de leadership dos seus dirigentes, começando a verificar-se um planning gap entre o planeado e a realidade, situação que requereu uma mudança de estratégia: a missão e os objetivos devem ser constantemente repensados para se gerar a melhor estratégia para a organização. No ano 2008, a empresa apresentou um Z-score superior ao limiar da falência provável, portanto, em termos futuros, a evolução previsível da empresa apontava inequivocamente para a continuidade. Como corolário da estratégia desenvolvida, o going concern e o Z-score apresentavam um equilíbrio saudável e permitiam ao auditor externo reforçar a sua convicção de continuidade.

No período de 2008 a 2012, a envolvente económica é caraterizada pela incerteza. Como não foram preventivamente analisadas as oportunidades e ameaças subjacentes à envolvente externa (de que se selecionam os fatores estratégicos externos) e observadas as forças e fraquezas da organização, de que se selecionam os fatores estratégicos internos, constata-se que a estratégia de crescimento por meio de nicho de mercado (construção e manutenção de equipamentos industriais de grandes empresas), materializadas em produtividade, tempo e concomitante redução dos custos, começava a não ter sucesso, situação que se refletia negativamente nas demonstrações de resultados e balanço, e o Z-score, como instrumento de monitorização, evidenciava indicadores preocupantes. 


\subsection{Demonstrações financeiras}

Apresentam-se as demonstrações financeiras da entidade.

Quadro 1 - Balanço da entidade de 2008 a 2012.

\begin{tabular}{|c|c|c|c|c|c|}
\hline \multicolumn{6}{|c|}{ Balanço (valores em euros) } \\
\hline & 2008 & 2009 & 2010 & 2011 & 2012 \\
\hline \multicolumn{6}{|l|}{ Ativo } \\
\hline Ativo não corrente & 2.536 .937 & 2.387 .774 & 4.917 .789 & 5.014 .641 & 4.875 .137 \\
\hline Ativo corrente & 14.445 .800 & 8.205 .765 & 9.260 .130 & 10.511 .409 & 8.728 .093 \\
\hline Total Activo & 16.982 .737 & 10.593 .539 & 14.177.919 & 15.526 .050 & 13.603 .229 \\
\hline \multicolumn{6}{|l|}{ Cap. Próprio + Passivo } \\
\hline \multicolumn{6}{|l|}{ Capital Próprio } \\
\hline Capital Social & 603.000 & 603.000 & 1.567 .800 & 2.508 .480 & 2.508 .480 \\
\hline Reservas & 1.278 .476 & 1.073 .206 & 1.181 .688 & 669.668 & 680.997 \\
\hline Resultado Líquido & 201.474 & 43.643 & 87.762 & 11.329 & -756.624 \\
\hline Total C. Próprio & 2.082 .950 & 1.719 .849 & 2.837.250 & 3.189 .477 & 2.432 .853 \\
\hline \multicolumn{6}{|l|}{ Passivo } \\
\hline Passivo não corrente & 915.417 & 779.183 & 3.191 .306 & 2.281 .291 & 2.063 .942 \\
\hline Passivo corrente & 13.984 .370 & 8.094 .507 & 8.149 .363 & 10.055 .283 & 9.106 .434 \\
\hline Total Passivo & 14.899 .787 & 8.873 .690 & 11.340 .669 & 12.336 .574 & 11.170.376 \\
\hline Total Pas. + Cap. Prop. & 16.982 .737 & 10.593 .539 & 14.177.919 & 15.526 .050 & 13.603 .229 \\
\hline
\end{tabular}

Fonte: Dados de uma empresa do setor metalúrgico. 
Quadro 2 - Demonstração de Resultados de 2008 a 2012.

\begin{tabular}{|c|c|c|c|c|c|}
\hline \multicolumn{6}{|c|}{ Demonstrações de Resultados (valores em euros) } \\
\hline & 2008 & 2009 & 2010 & 2011 & 2012 \\
\hline Vendas + PS & 30.502 .630 & 16.080 .979 & 21.098 .950 & 18.238 .490 & 13.500 .000 \\
\hline Ganhos/perdas subsidiárias & 0 & 32 & 0 & 1.278 & 5.000 \\
\hline Variação produção & -236.192 & 0 & 0 & 0 & 0 \\
\hline Trabalhos Prop. Entidade & 27.241 & 130.237 & 126.723 & 0 & 0 \\
\hline CMVMC & -4.405 .480 & -2.406 .388 & -4.391 .623 & -2.911 .558 & -2.041 .173 \\
\hline FSE & -20.157 .366 & -7.759 .962 & -8.548 .006 & -6.130 .206 & -5.959 .008 \\
\hline Gastos Pessoal & -4.520 .625 & -5.009 .240 & -6.436 .686 & -7.955 .701 & -5.019 .645 \\
\hline Imparidades & 19.122 & 1.764 & -778.203 & 0 & 0 \\
\hline Aumentos/reduções JV & 0 & 0 & 7.720 & -5.482 & 0 \\
\hline Outros rendimentos e ganhos & 100.025 & 222.989 & 34.263 & 90.885 & 39.070 \\
\hline Outros gastos e perdas & -129.742 & -245.680 & -114.450 & -199.893 & -87.132 \\
\hline $\begin{array}{l}\text { Resultado antes Depreciações, } \\
\text { Gastos Fin. e Impostos }\end{array}$ & 1.199 .614 & 1.014 .732 & 998.689 & 1.127 .812 & 437.112 \\
\hline $\begin{array}{l}\text { Gastos/ Reversões de } \\
\text { Depreciações }\end{array}$ & -364.730 & -376.419 & -360.202 & -440.215 & -450.000 \\
\hline Imparidades & 0 & 56 & -8.776 & 0 & 0 \\
\hline Resultado Operacional (RAJI) & 834.883 & 638.369 & 629.711 & 687.598 & -12.888 \\
\hline $\begin{array}{l}\text { Juros e Rendimentos Similares } \\
\text { Obtidos }\end{array}$ & 0 & 0 & 0 & 0 & 0 \\
\hline $\begin{array}{l}\text { Juros e Gastos Similares } \\
\text { Suportados }\end{array}$ & -388.432 & -447.407 & -450.414 & -564.407 & -693.736 \\
\hline RAl & 446.451 & 190.962 & 179.297 & 123.191 & -706.624 \\
\hline ISR & -244.978 & -147.319 & -91.535 & -111.862 & -50.000 \\
\hline Resultado Liquido do período & 201.474 & 43.643 & 87.762 & 11.329 & -756.624 \\
\hline
\end{tabular}

Fonte: Dados de uma empresa do setor metalúrgico. 


\subsection{Aplicação do modelo}

De acordo com a aplicação do modelo Z-Score, obtiveram-se os seguintes resultados:

Quadro 3 - Aplicação do modelo Z-Score ao balanço da entidade de 2008 a 2012.

\begin{tabular}{|c|c|c|c|c|c|}
\hline & 2008 & 2009 & 2010 & 2011 & 2012 \\
\hline Cap. Próprio & $2.082 .950,00$ & $1.719 .849,04$ & $2.837 .249,72$ & $3.189 .476,69$ & $2.432 .852,85$ \\
\hline Passivo & $14.899 .786,97$ & $8.873 .690,16$ & $11.340 .668,92$ & $12.336 .573,57$ & $11.170 .376,29$ \\
\hline Book Value & 0,14 & 0,19 & 0,25 & 0,26 & 0,22 \\
\hline $\begin{array}{l}\text { Capital } \\
\text { Social }\end{array}$ & $603.000,00$ & $603.000,00$ & $1.567 .800,00$ & $2.508 .480,00$ & $2.508 .480,00$ \\
\hline C. Circulante & $461.429,76$ & $111.258,57$ & $1.110 .767,52$ & $456.126,65$ & $-378.341,82$ \\
\hline Ativo Líquido & $16.982 .736,97$ & $10.593 .539,20$ & $14.177 .918,64$ & $15.526 .050,26$ & $13.603 .229,31$ \\
\hline Reservas & $1.479 .950,00$ & $1.116 .849,04$ & $1.269 .449,72$ & $680.996,69$ & $680.996,69$ \\
\hline RAJI & $648.349,16$ & $638.368,97$ & $629.711,07$ & $687.597,85$ & $-12.887,66$ \\
\hline Vendas & $30.502 .630,34$ & $16.080 .979,37$ & $21.098 .949,54$ & $18.238 .489,70$ & $13.500 .000,00$ \\
\hline & & & & \multicolumn{2}{|c|}{ Valores em euros } \\
\hline$x 1$ & 0,03 & 0,01 & 0,08 & 0,03 & $-0,03$ \\
\hline$x 2$ & 0,09 & 0,11 & 0,09 & 0,04 & 0,05 \\
\hline$x 3$ & 0,04 & 0,06 & 0,04 & 0,04 & 0,00 \\
\hline $\mathrm{x} 4$ & 0,14 & 0,19 & 0,25 & 0,26 & 0,22 \\
\hline$x 5$ & 1,80 & 1,52 & 1,49 & 1,17 & 0,99 \\
\hline Z-Score & 2,16 & 1,99 & 2,00 & 1,57 & 1,16 \\
\hline
\end{tabular}

Fonte: Dados de uma empresa do setor metalúrgico.

Graficamente, a evolução do resultado do modelo (Z) apresentase da seguinte forma:

Gráfico 1: Evolução do Resultado do modelo (Z) por ano, de 2008 a 2012.

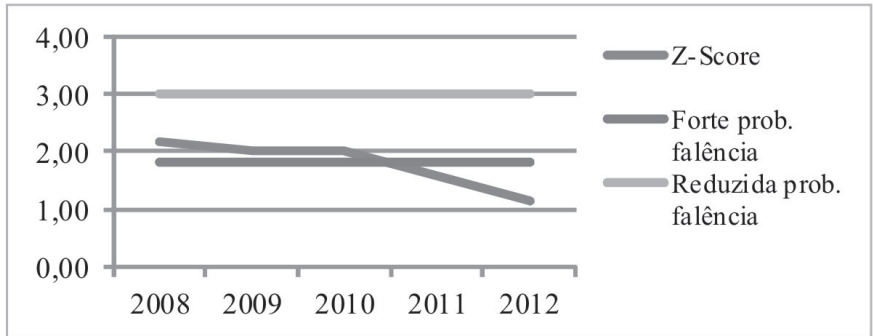

Fonte: Dados de uma empresa do setor metalúrgico. 
Como se verifica, entre o ano de 2008 e 2010, a entidade apresentou valores $(Z)$ superiores ao limiar de falência provável. Apesar de não serem ótimos, são valores que consideramos aceitáveis. De acordo com o modelo, para aquele período, a entidade evidencia uma situação de insucesso pouco provável: durante esses exercícios económicos, a continuidade da entidade nunca foi colocada em causa, como se veio a comprovar.

Para o ano de 2011, o valor obtido foi inferior ao limiar de insucesso provável. Apesar de existirem indicadores em clara degradação, o julgamento do órgão de gestão era favorável à continuidade da empresa. Vários fatores permitiram chegar a essa conclusão: as encomendas em carteira, a abertura da empresa ao mercado externo e a criação de uma filial em Moçambique, mercado de um rápido crescimento.

Prevê-se que, para o ano de 2012, os resultados sejam ainda mais gravosos, com o indicador em descida. Para que se percebam as causas dessa evolução, devemos analisar cada uma das variáveis do modelo de per si.

Gráfico 2: X1 (Fundo de Maneio / Ativo) por ano, de 2008 a 2012.

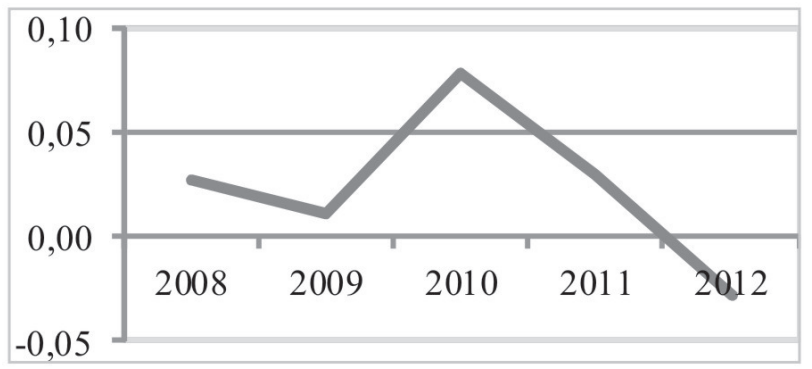

Fonte: Dados de uma empresa do setor metalúrgico.

$\mathrm{O}$ indicador anterior, sendo um indicador de equilíbrio financeiro de curto prazo, tenta refletir a capacidade de a entidade solver os seus compromissos, isto é, se tem ou não problemas de liquidez. O gráfico apresenta uma clara tendência negativa, com um cada vez maior problema de liquidez e de tesouraria, tal como já havíamos evidenciado. 
Gráfico 3: X2 (Reservas / Ativo) por ano, de 2008 a 2012.

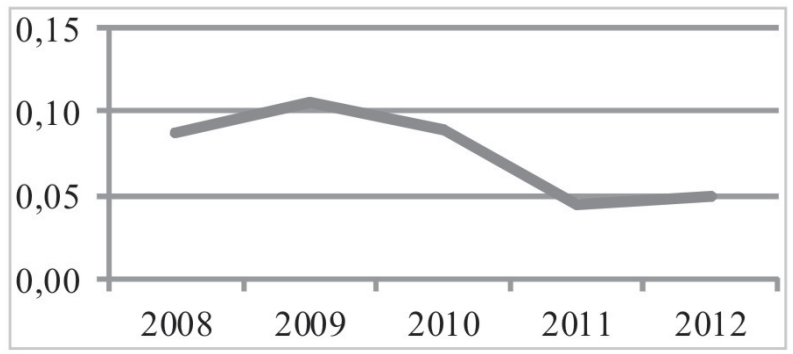

Fonte: Dados de uma empresa do setor metalúrgico.

Essa segunda variável, que relaciona as reservas (lucros retidos) com o seu ativo total, mostra a capacidade que a entidade tem em gerar resultados e mantê-los com o objetivo de serem reinvestidos. Como se verifica, essa capacidade é cada vez mais reduzida. Não é alheio o facto de terem existido distribuições de dividendos nem o facto de se ter aumentado o capital social por incorporação de reservas.

Nesse caso, o indicador é influenciado negativamente, apesar de não existir qualquer efeito real. Interessa referir que, neste caso, a entidade fica mais "desprotegida" no que concerne ao cumprimento do artigo $35^{\circ}$ do Código Português das Sociedades Comerciais, dado que o capital próprio fica com valores mais próximos do capital social, o que, existindo resultados negativos, como se prevê que possa acontecer, faz com que o primeiro passe a ser inferior ao segundo.

Ainda assim, essa empresa não tem problemas relacionados com esse assunto - perda de metade do capital -, o que não significa que tal situação não possa a vir ocorrer, caso a tendência não seja invertida. 


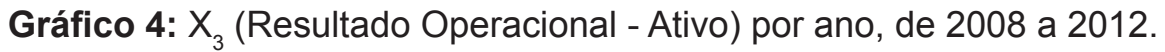

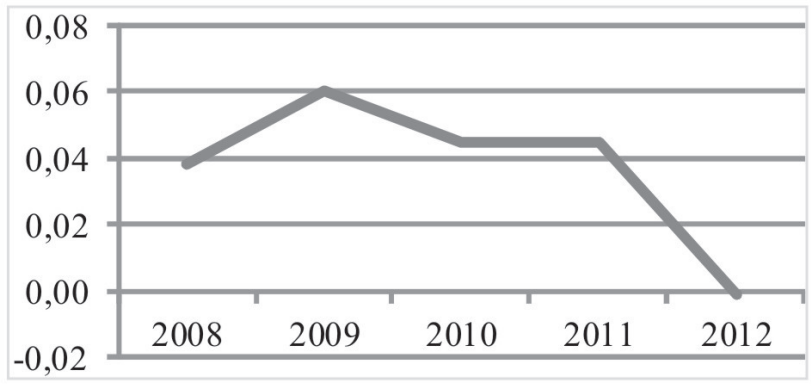

Fonte: Dados de uma empresa do setor metalúrgico.

Este rácio mede a rendibilidade do ativo e, como se verifica, apresenta também uma tendência negativa. Esta é grandemente influenciada pelo ano de 2012, que poderá ser negativo, o que significa que deverão ser adotadas medidas, como veremos mais adiante, para inverter esta tendência e assegurar a continuidade da entidade.

Gráfico 5: $\mathrm{X}_{4}$ (Valor da Empresa / Capitais Alheios) por ano, de 2008 a 2012.

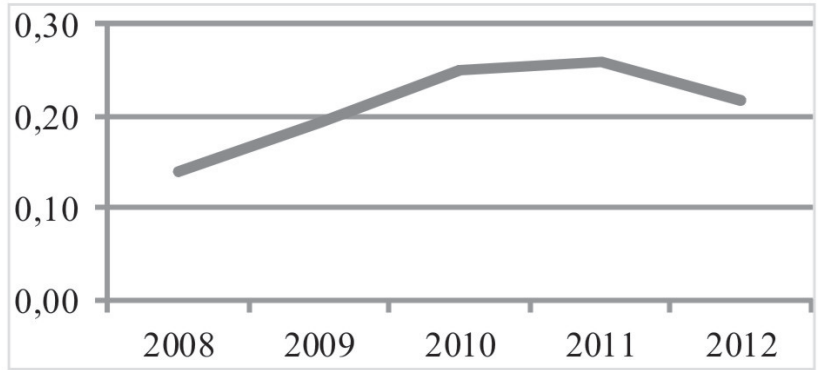

Fonte: Dados de uma empresa do setor metalúrgico.

Este rácio mede o grau de endividamento da entidade, relacionando o seu valor com os seus capitais alheios totais. Dado não se tratar de uma entidade cotada em bolsa, não conseguimos saber qual a sua capitalização bolsista, pelo que consideraremos o valor contabilístico da entidade (capital próprio). A entidade tem vindo a aumentar os seus 
capitais próprios, mantendo nos últimos anos o mesmo nível de passivo, pelo que a tendência é positiva. De qualquer modo, cerca de metade dos capitais alheios totais dizem respeito a financiamentos obtidos.

Essa é uma situação de risco, tendo em conta que a entidade suporta cada vez mais encargos financeiros e os próprios bancos tendem a ser cada vez mais exigentes na renegociação dos créditos existentes (por exemplo, contas correntes caucionadas ou descobertos bancários).

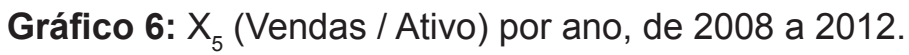

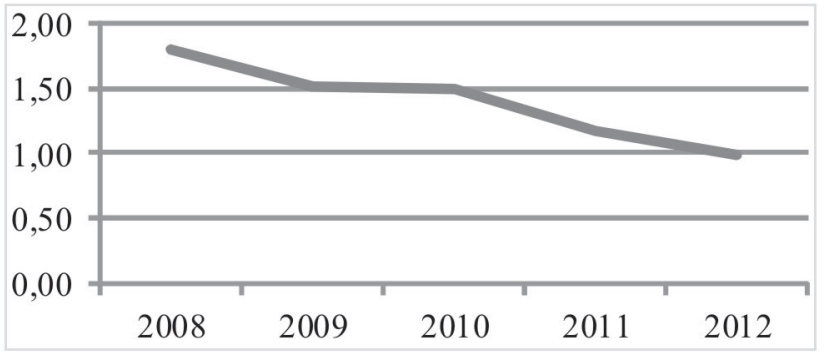

Fonte: Dados de uma empresa do setor metalúrgico.

Esse indicador relaciona as vendas com o ativo da entidade, permitindo aferir o grau de rotatividade deste face às vendas. Também apresenta uma tendência negativa, por via, essencialmente, da redução do volume de negócios da entidade nos últimos três anos analisados.

\subsection{Turnaround: 2012-2015}

Face a uma procura dos serviços prestados pela empresa, em contínua redução, como está evidenciado nas demonstrações financeiras históricas, a direção da empresa reavaliou a estratégia até ao horizonte temporal de 2015. Admitiu que os seus principais clientes (Cimpor, Somincor, Celuloses e outros), indústrias em maturidade, continuariam a operar tendencialmente a um menor nível de atividade. Essa constatação e a previsível redução do nível de atividade terão efeito no maior espaçamento temporal das manutenções, e, nesse contexto, a 
empresa em estudo tenderá a diminuir o seu breakeven, adequando-o à nova dimensão desejada.

Nesta altura, as técnicas de previsão quantitativa utilizadas na projeção dos fundamentos do negócio têm de ser complementadas com modelos qualitativos (estudos de mercado), tendo em conta a prudência exigida pela conjuntura: os modelos de previsão não valem senão pelos seus pressupostos. A estratégia competitiva da empresa, em função de um cenário prospetivo de estagnação, desenvolver-se-á através da modificação da sua estrutura orgânica e cultura empresarial, o que impõe o repensar da organização. Clarificadas as ideias do futuro estagnação no mercado interno e ameaças e oportunidades decorrentes -, a empresa passa a definir as estratégias necessárias, a partir de cenários pertinentes e prudentes.

Definido o que pode acontecer, a preparação e a avaliação das escolhas estratégicas para preparar as mudanças futuras permitem responder às seguintes questões: que posso fazer? Que quero eu fazer? Como fazer?

Elaborado o scanning environment, utilizando o benchmarking, e avaliado o risco inerente à estratégia eleita, a administração decidiu as seguintes estratégias para efetuar o turnaround:

- Estratégias de custos: redução da estrutura da empresa equilibrando-a com o novo nível de atividade.

- Estratégia de diversificação geográfica: aposta em novos mercados em desenvolvimento, em países africanos de expressão portuguesa.

- Estratégia de especialização: enfoque nas grandes empresas que realizam obras de manutenção de valores elevados.

Essas estratégias obrigaram a elaborar informação prospetiva para o triénio de 2013-2015, assente em pressupostos baseados nas estratégias anteriormente definidas. A sua implantação gerou programas, orçamentos e procedimentos, sujeitos a avaliação e controlo para monitorizar o strategic gap. 
Construíram-se, assim, as demonstrações de resultados previsionais, que facultam informação financeira prospetiva. Aplicámos o Z-score a esse tipo de dados, que terá por base a implementação da estratégia delineada, e observámos que os Z-scores conhecem uma evolução lenta, mas positiva, a partir do ano de 2013. O Z-score monitoriza de forma permanente, a implementação da estratégia e a sua reflexão nas demonstrações de resultados.

Assim, as medidas a implementar pelo órgão de gestão:

- Despedimento coletivo de 50-60 colaboradores - redução de custos 1.000.000€

- Adequação dos restantes gastos de funcionamento ao novo nível de atividade

- Aumento das vendas no mercado africano por via da subsidiária

De acordo com o descrito, os seus capitais próprios mantêm-se num nível elevado. Apesar de se prever que venham a reduzir, não se colocam em questão quaisquer problemas relacionados com o art. $^{\circ} 35$ do código das sociedades comerciais. Naturalmente que, não mudando o caminho a percorrer, provavelmente esse tipo de problemas irá surgir. Tendo em conta as previsões da empresa em análise para os próximos três anos, e tendo sido verificados os respetivos pressupostos, elaborámos as seguintes demonstrações financeiras: 
Quadro 4 - Balanço da entidade de 2012 a 2015.

\begin{tabular}{|c|c|c|c|c|}
\hline \multicolumn{5}{|c|}{ Balanço (valores em euros) } \\
\hline & 2012 & 2013 & 2014 & 2015 \\
\hline \multicolumn{5}{|l|}{ Ativo } \\
\hline Ativo não corrente & 4.875 .137 & 4.747.338 & 4.313 .480 & 3.978.271 \\
\hline Ativo corrente & 8.728 .093 & 8.634 .255 & 8.604 .103 & 8.500 .489 \\
\hline Total Activo & 13.603 .229 & 13.381.592 & 12.917 .583 & 12.478 .760 \\
\hline \multicolumn{5}{|c|}{ Cap. Próprio + Passivo } \\
\hline \multicolumn{5}{|l|}{ Capital Próprio } \\
\hline Capital Social & 2.508 .480 & 2.508 .480 & 2.508 .480 & 2.508 .480 \\
\hline Reservas & 680.997 & -75.627 & -73.857 & 104.291 \\
\hline Resultado Liquido & -756.624 & 1.770 & 178.148 & 428.110 \\
\hline Total C. Próprio & 2.432 .853 & 2.434 .623 & 2.612 .771 & 3.040 .881 \\
\hline \multicolumn{5}{|l|}{ Passivo } \\
\hline Passivo não corrente & 2.063 .942 & 2.022 .663 & 1.748 .236 & 1.718 .859 \\
\hline Passivo corrente & 9.106 .434 & 8.924.306 & 8.556 .577 & 7.719 .020 \\
\hline Total Passivo & 11.170 .376 & 10.946 .969 & 10.304 .812 & 9.437 .879 \\
\hline Total P.+Cap. Prop. & 13.603 .229 & 13.381 .592 & 12.917 .583 & 12.478 .760 \\
\hline
\end{tabular}

Fonte: Dados de uma empresa do setor metalúrgico. 
Quadro 5 - Demonstração de resultados da entidade de 2012 a 2015.

\begin{tabular}{|c|c|c|c|c|}
\hline \multicolumn{5}{|c|}{ Demonstrações de Resultados (valores em euros) } \\
\hline & 2012 & 2013 & 2014 & 2015 \\
\hline Vendas + PS & 13.500 .000 & 13.000 .000 & 13.500 .000 & 14.250 .000 \\
\hline Ganhos/perdas subsidiárias & 5.000 & 10.000 & 75.000 & 150.000 \\
\hline Variação produção & 0 & 0 & 0 & 0 \\
\hline Trabalhos Prop. Entidade & 0 & 0 & 0 & 0 \\
\hline CMVMC & -2.041 .173 & -2.210 .000 & -2.295 .000 & -2.422 .500 \\
\hline FSE & -5.959 .008 & -5.362 .500 & -5.805 .000 & -5.985 .000 \\
\hline Gastos Pessoal & -5.019 .645 & -4.204 .351 & -4.117 .500 & -4.389 .713 \\
\hline Imparidades & 0 & 0 & 0 & 0 \\
\hline Aumentos/reduções JV & 0 & 0 & 0 & 0 \\
\hline Outros rendimentos e ganhos & 39.070 & 37.623 & 37.623 & 36.229 \\
\hline Outros gastos e perdas & -87.132 & -83.905 & -83.905 & -80.797 \\
\hline $\begin{array}{l}\text { Resultado antes Depreciações, } \\
\text { Gastos Fin. e Impostos }\end{array}$ & 437.112 & 1.186 .867 & 1.311 .218 & 1.558 .220 \\
\hline Gastos/ Rev. de Depreciações & -450.000 & -437.898 & -394.108 & -354.697 \\
\hline Imparidades & 0 & 0 & 0 & 0 \\
\hline Resultado Operacional (RAJI) & -12.888 & 748.969 & 917.110 & 1.203 .523 \\
\hline $\begin{array}{l}\text { Juros e Rendimentos Similares } \\
\text { Obtidos }\end{array}$ & 0 & 0 & 0 & 0 \\
\hline Juros e Gastos Similares Suportados & -693.736 & -695.744 & -660.957 & -641.128 \\
\hline RAI & -706.624 & 53.225 & 256.153 & 562.395 \\
\hline ISR & -50.000 & -51.455 & -78.006 & -134.285 \\
\hline Resultado Líquido do período & -756.624 & 1.770 & 178.148 & 428.110 \\
\hline
\end{tabular}

Fonte: Dados de uma empresa do setor metalúrgico.

Aplicando novamente o modelo Z-Score, mas incluindo as previsões de 2013 a 2015, temos: 
Quadro 6 - Aplicação do modelo Z-Score ao balanço da entidade de 2012 a 2015.

\begin{tabular}{|lcccc|}
\hline & $\mathbf{2 0 1 2}$ & $\mathbf{2 0 1 3}$ & $\mathbf{2 0 1 4}$ & $\mathbf{2 0 1 5}$ \\
\hline Capital Próprio & $2.432 .852,85$ & $2.434 .623,24$ & $2.612 .770,85$ & $3.040 .880,86$ \\
Passivo & $11.170 .376,29$ & $10.946 .968,76$ & $10.304 .812,29$ & $9.437 .879,43$ \\
Book Value & 0,22 & 0,22 & 0,25 & 0,32 \\
Capital Social & $2.508 .480,00$ & $2.508 .480,00$ & $2.508 .480,00$ & $2.508 .480,00$ \\
C. Circulante & $-378.341,82$ & $-290.050,98$ & $47.526,28$ & $781.468,77$ \\
Activo Líquido & $13.603 .229,31$ & $13.381 .592,46$ & $12.917 .582,55$ & $12.478 .760,00$ \\
Reservas & $680.996,69$ & $-75.627,15$ & $-73.856,76$ & $104.290,85$ \\
RAJl & $-12.887,66$ & $748.969,02$ & $917.110,02$ & $1.203 .522,66$ \\
Vendas & $13.500 .000,00$ & $13.000 .000,00$ & $13.500 .000,00$ & $14.250 .000,00$ \\
\hline & & & Valores em euros \\
\hline x1 & $-0,03$ & $-0,02$ & 0,00 & 0,06 \\
x2 & 0,05 & $-0,01$ & $-0,01$ & 0,01 \\
x3 & 0,00 & 0,06 & 0,07 & 0,10 \\
x4 & 0,22 & 0,22 & 0,25 & 0,32 \\
x5 & 0,99 & 0,97 & 1,05 & 1,14 \\
\hline Z-Score & $\mathbf{1 , 1 6}$ & $\mathbf{1 , 2 6}$ & $\mathbf{1 , 4 3}$ & $\mathbf{1 , 7 4}$ \\
\hline
\end{tabular}

Fonte: Dados de uma empresa do setor metalúrgico.

Como se verifica, tendo em conta as medidas implementadas e eventos previstos, existe uma inversão clara nesse indicador, apresentando-se agora num sentido ascendente em todos os rácios. A entidade encontra-se já muito próximo do limiar inferior.

Gráfico 7: Evolução do Z-score até 2015.

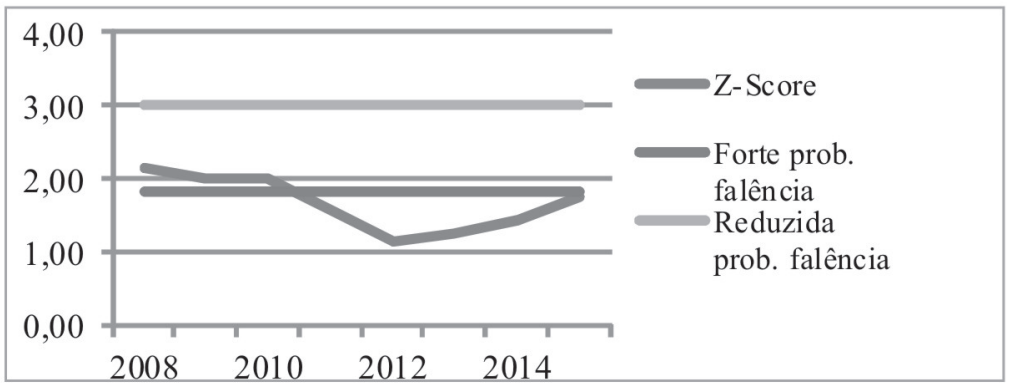

Fonte: Dados de uma empresa do setor metalúrgico. 
Desde o ano de 2011 que o modelo aplicado sugere uma possível rutura da entidade a breve trecho. $\mathrm{Na}$ realidade, isso não aconteceu. Tendo em conta a opinião da própria entidade, esse problema não se verifica. No entanto, essa situação não ocorrerá se a entidade se adequar ao seu novo nível de atividade. O mesmo se verifica quanto aos gastos de financiamento, que têm vindo a aumentar significativamente. Nesse caso, poderá não ser fácil reduzir os gastos, mas, caso exista uma boa reestruturação, não existirão necessidades de recurso a novos créditos, resolvendo a entidade este problema a médio prazo.

Até determinada altura, a entidade poderá sobreviver protelando pagamentos a fornecedores e até ao Estado, ou tentando fazer com que as próprias contas não reflitam a realidade. Tendo em conta que a entidade depende, em grande medida, do crédito bancário, a manipulação contabilística pode ser uma tentação, dado que, com resultados negativos, antever-se-á a não renovação de créditos existentes. O próprio relatório do auditor, tal como já foi referido, pode, eventualmente, precipitar esta situação.

Ainda assim, tendo em conta o exposto, a grande conclusão quanto a esse caso é que a continuidade deverá ser objeto de focagem. Como se verifica por meio do estudo apresentado, as condições económicas e financeiras dessa entidade têm vindo a degradar-se ao longo dos anos, e só com uma alteração no caminho traçado poderá subsistir, tal como constatámos. De acordo com as previsões decorrentes das medidas que se espera implementar, todos os indicadores invertem. É esse o objetivo da entidade que, iniciando agora essas novas mudanças, tentará prosseguir em continuidade.

Por essa razão, se os dados atuais fossem os dados da altura de aprovação de contas, a opinião seria, apesar do sugerido pelo modelo, que, mais do que prever falências, deve ser considerado um indicador da saúde da empresa concordar com o órgão de gestão quanto à continuidade da empresa, tendo, todavia, em conta que podem existir incertezas materiais. Dessa forma, caso aquelas fossem divulgadas, deveria ser incluído no relatório de auditoria um parágrafo de enfâse, e caso não fossem, uma reserva. 


\section{Considerações finais}

As escolas de posicionamento competitivo de Porter (1996), do desenvolvimento de competências distintivas (HAMEL; PRAHALAD, 1995), e da perspetiva estratégica de Godet (1997) e Mintzberg (1996) elegem a estratégia como um fator determinante de sobrevivência da empresa, orientação que, no caso vertente, foi seguida pelos administradores, ao definirem, até ao horizonte de 2015, uma estratégia que permitiu clarificar ideias relativamente ao futuro.

Essas estratégias motivaram a elaboração de informação prospetiva, seguindo a linha de orientação estratégica de Godet (1997), e construíram-se as demonstrações de resultados previsionais referentes ao horizonte temporal em análise, em termos flexíveis, consoante as sugestões da literatura internacional sobre o tema (GRANT, 2010).

O going concern está umbilicalmente ligado à estratégia definida pela empresa e requer que esta mantenha a sua capacidade competitiva, situação que hoje é discutida no âmbito da auditoria (BEAVER, 1966; MARQUES DE ALMEIDA, 2000).

Por sua vez, a técnica do Z-score insere-se no domínio da instrumentação contabilística, podendo ser considerada metaforicamente como um termómetro que nos ajuda a compreender a temperatura empresarial (ALTMAN; DANOVI; FALINI, 2013; ÖZDEMIR, 2014; SAMKIN; LOW; ADAMS, 2012).

Essa técnica é frequentemente utilizada e continua a merecer o interesse dos académicos, como se verifica na recente literatura académica internacional, sobretudo no contexto atual de incerteza em que vivem as empresas, e de forma singular as empresas portuguesas, que trabalham sob o efeito de uma crise interna e de uma crise internacional.

Assim, a estratégia, o going concern e o Z-score constituem um triângulo de fertilizações mútuas, que, utilizado prudentemente, pode desempenhar um papel importante quando está em causa o diagnóstico de continuidade da empresa. 
Ressalta do caso em estudo que a leitura das contas das empresas deve ser efetuada para além dos números agregados em mapas de síntese, e de acordo com as regras estritas da contabilidade. Hoje, deve privilegiar-se, como sucede no caso em estudo, uma visão sistémica da empresa (BELL; PEECHER; SOLOMON, 2005; MARQUES DE ALMEIDA, 2000; NORTON, 2012), que destaca a compreensão das vantagens estratégicas do cliente, a compreensão dos riscos que ameaçam a consecução dos objetivos da empresa e a capacidade de desenvolver previsões acerca dos pressupostos básicos subjacentes às demonstrações financeiras, que são normalmente preparadas no pressuposto da continuidade (IAS 1). O Z-score, como instrumento analítico de análise, foi determinante no caso em presença e revelou uma capacidade preditiva importante para o desenvolvimento de um mix de estratégia de turnaround: estratégia de custos, estratégia de diversificação e estratégia de especialização, que, de forma equilibrada, recuperaram a empresa e a sua continuidade, à semelhança das investigações desenvolvidas por Samkin, Low e Adams (2012), Aneja e Makkar (2013) e Özdemir (2014).

Por fim, pela dificuldade de avaliação do pressuposto da continuidade, principalmente em períodos de crise económica, e pela pressão que pode existir sobre os auditores quanto à modificação do relatório de auditoria, por motivos relacionados com a continuidade, é fundamental que se encontrem formas de corroborar a opinião. Os modelos preditivos de falências, tais como o Z-Score, cumprem essa função, não tanto por indicar de forma precisa a falha ou não da entidade, mas, essencialmente, por serem instrumentos de orientação do auditor quando aos problemas económicos e financeiros que possam vir a ocorrer.

Como foi analisado no caso real apresentado, o modelo pode sugerir uma falha eminente de uma entidade, mas, apesar disso, tendo em conta o conhecimento do negócio, a capacidade de governação do órgão de gestão e os seus planos contingentes para fazer face a situações de crise, a opinião do auditor pode assumir que o pressuposto da continuidade está bem aplicado. 
Por fim, como contribuições do artigo destacamos: a interligação entre a estratégia, o going concern e os modelos de previsão de falências; a ênfase colocada nas demonstrações financeiras apresentadas pela empresa, mais como produto de uma estratégia e menos como alinhamento de números para efeitos de prestação de contas; e a aplicação do modelo a um caso concreto.

\section{Referências}

ALTMAN, E. I.; Danovi, A.; Falini, A. Z-Score Models' Application to Italian Companies Subject to Extraordinary Administration. Journal of Applied Finance, South Florida, v. 23, n. 1, p. 128-137, 2013.

ALTMAN, E. I.; MCGOUGH, T. P. Evaluation of a company as a going concern. Journal of Accountancy, [S.I.], v. 6, p. 50-57, December 1974.

ANEJA, R.; MAKKAR, A. A Comparative Study of Book Value Insolvency of Indian Commercial Banks: An Application of

Z-ScoreModel. IUP Journal of Financial Risk Management, Telangana, India, v. 10, n. 2, p. 19-29, June 2013.

ANJUM, S. Business Bankruptcy Prediction Models: A Significant Study of the Altman's Z-Score Model. Asian Journal of Management Research, [S.I.], v. 3, n. 1, p. 212-219, Aug., 2012.

AUCHTERLONIE, D. L. A Paen to the Z-Score and Its Commercial Bankruptcy Prediction. The Journal of Lending \& Credit Risk Management, Chicago, v. 80, n. 1, pp. 50-52, Sept. 1997.

BEAVER, W. H. Financial, Ratios as Predictor of Failure. Journal of Accounting Research, Chicago,v. 4, p. 71-111, Oct., Spring 1966.

Bell, T.; Peecher, M.; Solomon, I. The 21st Century Public-Company Audit: Conceptual Elements of KPMG's Global Audit Methodology. New Jersey: KPMG: Montvale, 2005. 
BUENO CAMPOS, E. Dirección Estratégica de la Empresa: metodología, técnicas y casos. Madrid: Pirámide, 1996.

CHAO, H. Y. The Value of Style Rotation Strategies in Emerging Asian Market. [S.I] ProQuest UMI Dissertation Publishing, 2012. 202p.

DICKSEE, L. Auditing-A Practical Manual for Auditors. New York: Arno Press, 1905.

ERICKSON, F. Qualitative methods in research on teaching. In: WITROCK, M.C. Handbook of research on teaching. New York: Macmillan, 1986. p.119 - 161.

ESTADOS UNIDOS DA AMÉRICA. Public Law 107-204, JULY 30, 2002. Sarbanes Oxley Act, Dispinível em: <https://www.sec.gov/ about/laws/soa2002.pdf >. Acesso em: 02 jan. 2016.

FAYOL, H. Administration Industrielle et Générale. Paris : Dunod, 1916.

GODET, M. Manuel de Prospective Stratégique: Tomo II, L'art et la Méthode. Paris : Dunod, 1997.

GRANT, R. M. Contemporary Strategy Analysis: text and cases. 7. ed. West Sussex: John Wiley \& Sons, 2010.

HAMEL, G.; PRAHALAD, C. K. Competir pelo futuro. Rio de Janeiro: Campus, 1995.

Hawkins, T. G.; Nissen, M. E.; Rendon, R. G. Leveraging Strategic Sourcing and Knowledge Management to improve the acquisition of Knowledge based Services. Journal of Public Procurement, Florida, v. 14, n. 2, p. 215-251, May/ Set., 2014.

HEIZENBERG, W. K. The Physical Principles of the Quantum Theory. Courier Dover Publications, 1949.

HITT, M. A.; IRELAND, D.; HOSKISSON, R. E. Strategic Management: Competitiveness and Globalization Cases. 7. ed. Thomson, 2007. 
HITT, M. A.; IRELAND, R. D.; HOSKISSON, R. E. The Management of Strategy: concepts and cases. 8. ed. Australia: South Western, 2009.

IIASB. IAS 1 - Apresentação de Demonstrações Financeiras, Manual do Revisor Oficial de Contas. Disponível em: <http://www. manualdoroc.com>.Acesso em: 06 set. 2012

IJIRY, Y. Theory of Accounting Measurement. Sarasota, FL: American Accounting Association, 1975.

IFAC. ISA 220 - Controle de Qualidade para uma Auditoria de Demonstrações Financeiras, Manual do Revisor Oficial de Contas. Disponivel em: <http://www.manualdoroc.com/>. Acesso em: 06 set. 2012

IFAC. ISA 240 - As Responsabilidades do Auditor Relativas a Fraude numa Auditoria de Demonstrações Financeira, Manual do Revisor Oficial de Contas. Disponível em: <http://www.manualdoroc.com>. Acesso em: 06 set. 2012

IFAC. ISA 315 - Identificar e Avaliar os Riscos de Distorção Material Através do Conhecimento da Entidade e do Seu Ambiente, Manual do Revisor Oficial de Contas. Disponível em: <http://www. manualdoroc.com>. Acesso em: 06 set. 2012 IIASB. ISA 540 - Auditar Estimativas Contabilísticas, Incluindo Estimativas Contabilísticas de Justo Valor e Respectivas Divulgações, Manual do Revisor Oficial de Contas. Disponível em: <http://www. manualdoroc.com>. Acesso em: 06 set. 2012

KILPATRICK, J. Editorial. Journal for Research in Mathematics Education, [S.I], v. 18, n. 4, p. 98, Mar, 1998.

LAFLEY, A.G et al. Bringing Science to the Art of Strategy. Harvard Business Review, Massachusetts, [S.I], v. 90, n. 9, p. 51-59, Sept. 2012.

LEWIS, L. D. The Power of Accounting: what the numbers mean and how to use them. 1. ed. London: Routledge, 2012. 
LITTLETON, A. C. Accounting Evolution to 1900. Glasgow Lanarkshire: Russel and Russel, 1966.

MARQUES DE ALMEIDA, J. J. Auditoria Previsional e Estratégica. Lisboa: Vislis Editores, 2000.

MINTZBERG, H. The Strategy Process Concepts, Contexts, Cases. 3. ed. London: Prentice Hall International, 1996.

NORTON, D. The Executive's Guide to Financial Management: improving risk, strategy, and financial performance. [S.I]: Palgrave macmillan, 2012.

ÖZDEMIR, F. S. Turkish Uniform Accounting System and Applicability of Altman Z Score Models in the Context of Public and Private Companies. Ege Academic Review, Bornova/Izzmir, Turkeyv, v.14, n. 1, p. 147-162, 2014.

PARASCHIVESCU, A. O.; CĂPRIOARĂ, F. M. Strategic Quality Management. Economy Transdisciplinarity Cognition, Bacau, Romania, v. 17, n. 1, p. 19-27, 2014.

PATON, V. A. Accounting Theory. Chicago: Accounting Studies Press Ltd., 1962.

PONTE, J. Estudos de caso em educação matemática. Bolema, [S.I]. v. 25, p. $105-132,2006$.

PORTER, M. E. The Five Competitive Forces That Shape Strategy. Harvard Business Review, Massachusetts, [S.I], v. 86, n. 1, p. 78-93, Jan. 2008.

PORTER, M. O que é a estratégia. 1996. Disponivel em: <http://www3. pucrs.br/pucrs/files/adm/asplam/estrategia.pdf>. Acesso em: 02 jan. 2015.

RÃSCOLEAN, I.; DOBRA, R.; SLUSARIUC, G. C. Predictive Analysis Software for Modeling the Altmanz-Score Financial Distress Status of 
Companies. Annals of the University of Petrosani Economics, v. 12, n. 3, p. 231-240, 2012.

STERLING, R. R. The Going Concern - An Examination. The Accounting Review, [S.I], v. 43, n. 3, p. 481-502, July 1968.

TAYLOR, D. H.; GLEZEN, G. W. Auditing Integrated Concepts and Procedures. 6. ed. John Wiley \& Sons Inc, 1994.

VAZ FREIXO, H. J. Metodologia Científica: fundamentos métodos e técnicas. 3. ed. Lisboa: Instituto Piaget, 2011.

VENDERBECK, E. J. Principles of Cost Accounting. 16. ed. SouthWestern College Pub, 2012.

YIN, R. K. Case Study Research. Design and Methods. Newbury Park: Sage, 1988.

ZMIJEWSKI, M. Methodological Issues Related to Estimation of Financial Distress Prediction Models. Journal of Accounting Research, Chicago, v. 22, p. 59-82, Dec., 1984.

Artigo recebido em: 23/01/2015

Aprovado em: 04/11/2015 